\title{
Markers of Preclinical Atherosclerosis and their Clinical Relevance
}

\author{
Pavel Poredos*
}

Department of Vascular Disease, Ljubljana University Medical Centre, Zaloska Cesta 7, 1000 Ljubljana, Slovenia

\begin{abstract}
The estimation of risk for atherosclerotic and cardiovascular events based only on the presence of classical risk factors is often insufficient. Therefore, efforts have been made to find markers that indicate the presence of preclinical disease in individual subjects like blood markers of atherosclerosis and preclinical deterioration of the arterial wall.

Elevated levels of several inflammatory mediators have been found in subjects with atherosclerosis. Prospective epidemiological studies have found increased vascular risk in association with increased basal levels of cytokines, the cell adhesion molecules P-selectin and E-selectin; and acute-phase reactants such as high sensitive C-reactive protein (hsCRP), fibrinogen, and serum amyloid A. For clinical purposes, the most promising inflammatory biomarker appears to be hsCRP.

In the last decade, markers of plaque stability and unstable coronary artery disease have been sought such as myeloperoxidase, soluble CD40 ligand, pregnancy-associated plasma protein A, free fatty acids and placental growth factor.

Further, markers of endothelial dysfunction (ED), like circulating molecules as well as indicators of functional deterioration of the arterial wall, that represent a common denominator of harmful effects of risk factors on the vessel wall were identified. It was show that endothelial dysfunction is closely related to different risk factors of atherosclerosis, and to their intensity and duration.

Measurement of the intima-media thickness (IMT) using high resolution B-mode ultrasonography has emerged as one of the methods of choice for determining the anatomic extent of preclinical atherosclerosis and for assessing cardiovascular risk. A strong correlation between carotid IMT and several cardiovascular risk factors was shown and it has also been found to be associated with the extent of atherosclerosis and end-organ damage of high risk patients.

Determination of markers of preclinical atherosclerosis could influence the decision of a clinician to intervene with medication and to use more aggressive treatment of risk factors in primary prevention, and in patients with atherosclerotic disease.
\end{abstract}

Keywords: Circulating markers, endothelial dysfunction, intima media thickness.

\section{INTRODUCTION}

The first clinical manifestation of cardiovascular disease often arises in the stage of well-advanced atherosclerosis. However, functional and morphological deterioration of the arterial wall occurs during a presumably long subclinical lag (silent) phase that usually take more decades. As the maximal potential for prevention and reversibility of atherosclerosis would be expected by intervention at an early stage of the disease, beside identification of risk factors of atherosclerosis, the detection of early morphological and functional changes of the arterial wall is of utmost importance for prevention of progression of atherosclerosis and cardiovascular (CV) events [1]. Estimation of the risk of $\mathrm{CV}$ events based only on the presence of classical risk factors is commonly insufficient and classical risk factors can be identified in only $50 \%-70 \%$ of subjects with developed atherosclerosis. Therefore, efforts have been made to find other non-classical markers of atherosclerosis and to

*Address correspondence to this author at the Department of Vascular Diseases, Ljubljana University Medical Centre, Zaloska cesta 7, 1000 Ljubljana, Slovenia; Tel: + 386152280 32; Fax: + 386152280 70;

E-mail: pavel.poredos@kclj.si detect atherosclerotic changes in their preclinical phases. Further, preclinical markers of atherosclerosis enable identification of individuals in whom the atherosclerotic process is already present. Consequently, in recent years, research on atherosclerosis has been mainly focused towards identifying markers of early preclinical atherosclerosis. The most frequently studied are circulating markers and markers of early arterial wall alteration such as arterial wall thickening and endothelial dysfunction.

\section{CIRCULATING MARKERS}

Recently, many investigators have focused on the determination of circulating - blood markers of atherosclerosis. There are a number of markers found in blood that indicate the presence of the atherosclerotic process and represent risk for the development of $\mathrm{CV}$ events [2]. Further, they may be used for identifying subjects predisposed to plaque disruption and vascular obstruction. Recent investigations have indicated that increases in biomarkers upstream of markers of necrosis may have not only the utility to identify patients with acute coronary syndrome, but to recognize patients with higher risk of complications [3]. 
Inflammatory Markers of Atherosclerosis and Risk of Cardiovascular Events

As atherosclerosis is recognized as a chronic inflammatory disease in patients with the atherosclerotic process, increased levels of systemic inflammatory markers are expected. Elevated levels of several inflammatory mediators among apparently healthy men and women have proven to have predictive value for future vascular events [4]. In particular, prospective epidemiological studies have found increased vascular risk in association with increased basal levels of cytokines such as IL-6 and TNF- $\alpha$, cell adhesion molecules such as soluble ICAM-1, P-selectin Eselectin, and downstream acute-phase reactants such as high sensitive C-reactive protein (hsCRP), fibrinogen, and serum amyloid A [5].

For clinical purposes, the most promising inflammatory biomarker appears to be hsCRP, a classical acute-phase marker. In addition to providing downstream integration of overall cytokine activation hsCRP has several direct effects that may affect vascular disease progression. These include an ability to bind and activate complement, induce expression of several cell adhesion molecules as well as tissue factor, mediate LDL uptake by endothelial macrophages, induce monocyte recruitment into the arterial wall, and enhance production of monocyte chemotactic protein 1 (MCP-1).

Population-based studies have demonstrated that baseline hsCRP levels predict future cardiovascular events. High sensitive CRP testing may thus have a major adjunctive role in the global assessment of cardiovascular risk [6]. In addition to providing a simple method to assess low-grade inflammation and improve global risk prediction, hsCRP screening may also provide a novel method of targeting statin therapy, particularly in the primary prevention of myocardial infarction and stroke. Both experimental and clinical outcome data now support the hypothesis that statins, in addition to being potent LDL-lowering agents, also attenuate plaque inflammation and influence plaque stability [7]. The JUPITER trial demonstrated that not only individuals with high LDL cholesterol but also those with low LDL-C and high levels of hsCRP may benefit from statin therapy in the primary prevention of CV events $[8,9]$.

The utility of hsCRP as a screening test has been evaluated by numerous studies and hsCRP has been shown to reclassify patients in a global risk prediction algorithm who are classified as low-, moderate- or moderately high risk by the Framingham risk score (FRS). The Reynolds risk score was developed recently and includes hsCRP, family history and traditional risk factors in its risk prediction model [10]. Using the Reynolds risk score, many patients at intermediate risk (5\% to $20 \%$ risk of a CV event over the next decade) by FRS are reclassified into higher or lower risk categories. However, a meta-analysis of 22 prospective studies found that hsCRP contributed less than expected to risk prediction after adjusting for other risk factors.

Another indicator of inflammation response is fibrinogen, which promotes platelet aggregation and fibrin clot deposition and has also been shown to be elevated in individuals who are at risk of or who have coronary heart disease and cerebrovascular disease [11].
Interleukins are considered to be players in the chronic vascular inflammatory response that is typical of atherosclerosis. Thus, the expression of pro-inflammatory interleukins in their receptors has been demonstrated in atheromatous tissue. In vitro studies have confirmed the involvement of various interleukins in pro-atherogenic processes, such as the up-regulation of adhesion molecules on endothelial cells, the activation of macrophages, and smooth muscle cell proliferation [12]. Furthermore, studies in mice deficient or transgenic for specific interleukins have demonstrated that, whereas some interleukins are indeed intrinsically pro-atherogenic, others may have antiatherogenic qualities [13].

The serum levels of several of these cytokines have been found to be positively correlated with coronary artery disease (CAD) and its sequelae. Cytokines may represent the risk of $\mathrm{CV}$ events also in patients without classical risk factors [14].

\section{MARKERS OF PLAQUE STABILITY}

In the last decade, markers of plaque stability and unstable coronary artery disease have been sought: myeloperoxidase, metalloproteinase-9, soluble CD40 ligand, pregnancy associated plasma protein $\mathrm{A}$, ischaemia modified albumin, free fatty acids and placental growth factor.

\section{Myeloperoxidase}

Myeloperoxidase is a haemoprotein stored in the azurophilic granules of polymorphonuclear neutrophils and macrophages. Myeloperoxidase is released into the extracellular fluid and in circulation during inflammatory conditions. This enzyme has been implicated in the oxidation of lipids contained within LDL. Myeloperoxidase plays an important role in the pathogenesis of the destabilization of coronary artery disease, leading to acute coronary syndrome (ACS) [15]. Infiltrating macrophages and neutrophilis releasing myeloperoxidase participate in the transformation of stable coronary artery plaques into unstable lesions. Metalloproteinases (MMPs) and metal-independent myeloperoxidase degrade the collagen layer that protects atheromas from erosion or abrupt rupture. As a result, plaques that have been highly infiltrated with macrophages releasing MMPs are vulnerable to erosion or rupture, precipitating events leading to ACS.

Different clinical studies examined the role of myeloperoxidase as a marker of risk for ACS. In the CAPTURE trial, myeloperoxidase concentration was measured in 1090 patients with ACS. The death and MI rates were determined at 6 months of follow-up and the adjusted hazard ratio based on myeloperoxidase concentration was 2.25. The results were particularly impressive in patients with undetectable cardiac troponin $\mathrm{T}$, where the hazard ratio was 7.48 [16]. In the study of 604 sequential patients exhibiting chest pain, Brennan et al. demonstrated a progressive increase in odds ratios for major adverse events at 30 days and 6 months with each quartile increase in myeloperoxidase concentration [17]. Therefore, myeloperoxidase was accepted as a marker of plaque instability.

A study from the AetheroGene investigators on 1127 patients with either stable or unstable CAD reported that values of metalloproteinases were related to future 
cardiovascular death. Those in the highest quartile had a significantly increased risk of cardiac death whether in the stable or unstable group [18].

Of special interest has been the possibility that MMP concentrations might be helpful in evaluating the remodelling that occurs after MI. Recently, Squire et al. have reported their initial experience relating blood concentrations of MMP-9 and TIMP-1 to changes in ventricular function over a 6-week period in patients with ST-elevation myocardial infarction. MMP-9 manifested a biphasic pattern with peaks at 1 and 3 days and a nadir at day 2. Further, increased concentrations of MMP-9 were associated with larger ventricles and higher amino-terminal brain natriuretic peptide concentrations [19].

Therefore preliminary studies suggest that MMP may be of value in evaluating the prognosis of patients with CAD and after acute coronary events. However, more rigorous corrections with other biomarkers and clinical variables are necessary before any firm suggestion can be made.

\section{Soluble CD40 Ligand (sCD40L)}

Circulating sCD40L is derived largely from activated platelets and has biological activity that can trigger an inflammatory reaction in vascular endothelial cells by the secretion of cytokines and chemokines. SCD40L forms interact with the CD40 receptor molecule, which is present not only on B cells but also on monocytes, macrophages, endothelial and smooth muscle cells in atheromas, leading to the release of matrix MMPs and subsequent destabilization of the plaque. The release of tissue factor then activates platelets, which produce more sCD40L and perpetuate the inflammatory and prothrombotic environment in the vasculature [20].

Increased $\mathrm{SCD} 40 \mathrm{~L}$ concentrations have been demonstrated in inflammatory disorders, e. g. autoimmune diseases, multiple sclerosis, and inflammatory bowel disease, as well as in stroke, hypercholesterolemia, and diabetes. Up-regulation of the sCD40L system may play a pathogenic role also in triggering ACS [21].

In a cohort of 195 ACS cases and matched controls from the OPUS-TIM116 trial, the median SCD40L concentration was significantly higher in patients than in controls. In this study, increased $\mathrm{SCD} 40 \mathrm{~L}$ was associated with a higher risk for future recurrent cardiac events, and in combination with cardiac troponin, it improved risk stratification for death and MI [22-24].

In the CAPTURE study of unstable refractory angina, increased $\mathrm{SCD} 40 \mathrm{~L}$ concentrations were associated with a higher incidence of cardiac events in patients on placebo [16].

Thus, preliminary data demonstrate promise for commercialization of $\mathrm{sCD} 40 \mathrm{~L}$ as an independent biomarker of risk and prognosis.

\section{Unbound Free Fatty Acids}

Most serum-free fatty acids (FFAs) are bound to albumin, with only a small amount of the total unbound FFAs present as the soluble form. On the basis of preliminary findings, FFA concentration, rather than total
FFAs, may provide a sensitive guide to the pathophysiology of underlying coronary disease. In patients with ACS, the concentration of FFAs in circulation increases before traditional markers of myocyte necrosis [25].

Preliminary data suggest that in patients exhibiting ischaemic symptoms, monitoring of plasma FFAs may provide an early indication of cardiac ischaemia. Additional trials are needed to fully evaluate the true potential of this biomarker [26].

\section{Placental Growth Factor}

Placental growth factor (PlGF) is one of the family of platelet-derived proteins that function as potent chemoattractants for monocytes and are involved in the regulation of vascular endothelial growth. It was found in different tissues including thyroid, placenta, and lung. The biological functions of PlGF are incompletely understood, but they appear to primarily involve initiation of the inflammatory process, which includes the recruitment of circulating macrophages into atherosclerotic lesions, stimulation of smooth muscle cell growth, and up-regulation of both tissue necrosis factor and MCP-1 by macrophages [27].

Particular interest in PlGF has been sparked by its proposed role as a principal instigator of atherosclerotic plaque instability. Compelling data have shown that inhibiting the actions of PlGF suppressed plaque instability and coronary heart disease [28]. Thus, PIGF is not merely a risk marker but also a disease marker, and may represent a new therapeutic target for mitigating the disease process behind ACS.

In the CAPTURE cohort PIGF was shown to be an independent predictor of death or MI at 30 days, whereas increased CRP was not [29]. Further, plasma PIGF measurements have been shown to be an independent biomarker of adverse outcome in patients with suspected ACS. Plasma PlGF appears to extend the predictive and prognostic information gained from traditional biomarkers of necrosis, platelet activation, and systemic inflammation. PlGF is increased in ACS, regardless of the troponin concentration.

Therefore, PIGF appears to have great potential as an independent biomarker for plaque disruption, ischaemia and thrombosis. Larger trials are forthcoming and may aid in the possible commercialization of PlGF.

\section{ENDOTHELIAL DYSFUNCTION}

The vascular endothelium is an important regulatory organ in maintaining cardiovascular homeostasis. Normal endothelial function includes control over thrombosis and thrombolysis, platelets and leukocyte interaction with the vessel wall, and regulation of vascular tone and smooth muscle cell proliferation. One of the most important regulatory and vasoactive substances produced by endothelial cells is nitric oxide (NO). Nitric oxide modulates vascular tone, and inhibits the interaction between blood cells and the vessel wall (Fig. 1) [30].

Nitric oxide is a transient molecule produced from the amino acid arginine by nitric oxide synthesis (NOS) 


\section{ENDOTHELIAL FUNGTION}

Endothelial Cells Regulate Function and Structure of the Vessel Wall

\begin{tabular}{lll}
\hline Produce & Modulate & Provide \\
\hline - Vasoactive substances & - Vascular smooth & •A selectively \\
- Coagulation and & muscle cell & permeable \\
fibrinolytic mediators & function, & barrier between \\
- Growth factors and inhibitors & proliferation and & circulation \\
& migration & and vessel wall \\
\hline
\end{tabular}

\section{ENDOTHELLAL DYSFUNCTION}

Hypertension Diabetes Smoking LDL Homocysteine Estrogen
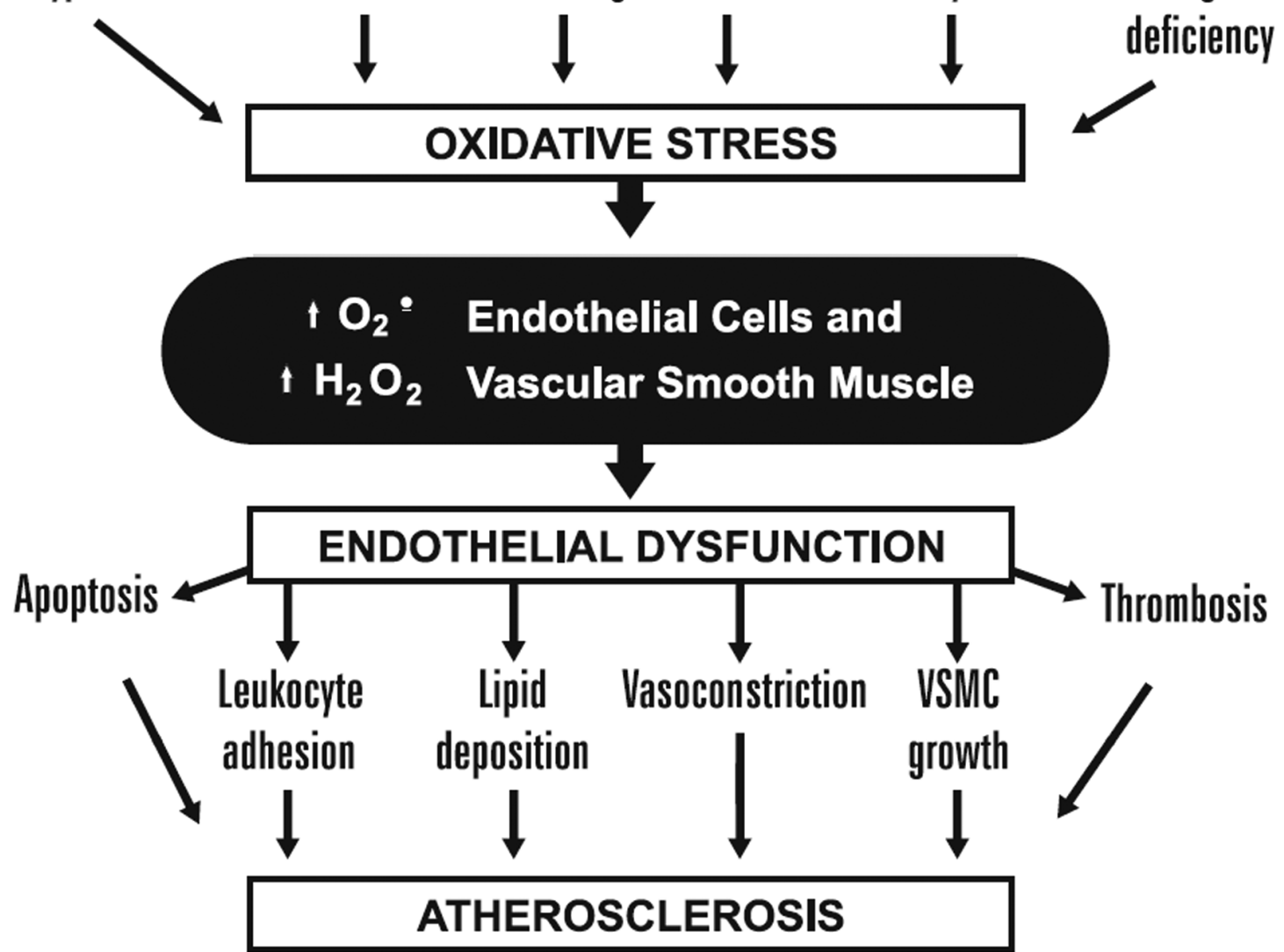
enzymes. Neuronal (nNOS) enzymes are crucial for signalling neuroprotection. Nitric oxide produced by the endothelial NOS synthase (eNOS) is a fundamental determinant of cardiovascular homeostasis: it regulates vascular tonus and systemic blood pressure, vascular remodelling and angiogenesis. Its release is continuous and is dependent on shear stress, generated by the streaming blood on the endothelial cells. Shear stress mediates phosphorylation of eNOS through activating the phosphatidylinositol 3-kinases (PI3-kinases) pathway which activates eNOS and leads to increased production of NO. Thus, phosphorylation of eNOS by PI3k/Akt pathway replenishing cellular tetrahydrobiopterin, a co-factor of eNOS, increasing transcription and activity of eNOS and leading to increased bioavailability of NO [31, 32]. Risk factors of atherosclerosis increase oxidative stress with producing free oxygen radicals which inactivate $\mathrm{NO}$ and inhibit PI3k/Akt pathway.

The so-called inducible (iNOS) enzyme represents NOS isoform found to be expressed in variety of immunomodulatory cells after their stimulation with inflammatory cytokines [33]. Inducible NOS appears to be expressed in some epithelial cells in the lungs, in gastrointestinal tract as well as in other cell types following their immunoactivation. Inducible $\mathrm{NO}$ is produced "ondemand" in high levels to help to kill tumour cells, bacteria and viruses. However, excess of NO production by iNOS can cause inflammation, rheumatoid arthritis, inflammatory bowel disease, immuno-type diabetes, stroke, and cancer. Excessive release of NO may also be responsible for hypotension in patients with sepsis and anaphylaxis [34].

Endothelial dysfunction is characterized by an imbalance between relaxing and contracting factors, between procoagulant and anticoagulant substances and between proinflammatory mediators [30]. Because healthy endothelium plays a central role in cardiovascular control, it follows that endothelial damage may contribute to disease states characterized by vasoconstriction, inflammation, excessive thrombus formation, leukocyte adhesion to vessel walls, hypertension, and finally atherosclerosis (Fig. 1).

\section{Indicators of Endothelial Dysfunction}

As endothelial dysfunction is a key underlying factor in the atherosclerotic process, markers of endothelial abnormalities have been sought, particularly those involving disturbed endothelium-dependent vasomotion or related circulating cellular products.

\section{CIRCULATING MARKERS OF ENDOTHELIAL DAMAGE}

Increased markers of endothelial function such as endothelin, adhesion molecules, von Willebrand factor and others indicate hyperactivity of endothelial cells or are the result of their damage, and most probably represent a common denominator of harmful effects of different risk factors on the vessel wall. Elevated levels of markers of endothelial function are predictive of the risk, presence, and severity of atherosclerotic vascular disease in an individual subject.

\section{Endothelin-1}

Endothelin-1 is an endothelium-derived peptide that has powerful vasoconstrictor properties. Increased levels of endothelin-1 have been demonstrated in conditions associated with endothelial dysfunction, such as atherosclerosis, hypercholesterolemia, and cigarette smoking. Furthermore, oxidized LDL has been shown to stimulate endothelin-1 production and secretion. Injury to vascular endothelial cells probably triggers the release of endothelin-1, and therefore it has been suggested that the levels of circulating endothelin in plasma may represent a marker of endothelial dysfunction - the earliest measurable functional deterioration of vessel wall in atherogenesis [35-37].

\section{Von Willebrand Factor}

Von Willebrand factor (vWF) is a glycoprotein synthesized mainly by vascular endothelial cells. It has an important function in haemostasis, participating in the coagulation and in the formation of platelet plugs at the sites of endothelial damage. Von Willebrand factor is elevated in patients with hypercholesterolemia, and reduction of total cholesterol has been shown to be associated with a reduction in $\mathrm{vWF}$ levels [38]. Its levels are elevated in situations characterized by vascular damage with denudation of the endothelium. Further, it has been suggested that injured endothelial cells leak $\mathrm{vWF}$, leading to increased plasma levels. Recent studies have shown that elevated levels of vWF may be predictive of recurrent cardiovascular events in patients with known cardiovascular disease [39-41].

\section{Tissue Plasminogen Activator and Plasminogen Activator Inhibitor-1}

Tissue plasminogen activator (t-PA) is a protein released by endothelial cells. It activates the reaction in which plasminogen is converted to plasmin. Tissue plasminogen activator regulates the fibrinolytic activity of blood in balance with plasminogen activator inhibitor-1 (PAI-1), another product of endothelial cells, serving as a primary inhibitor of t-PA. These circulating vascular endothelial markers are elevated in hypercholesterolemia [42]. Furthermore, prospective studies have shown that a high concentration of t-PA antigen in healthy men may be associated with a subsequent risk of myocardial infarction and stroke [43]. Data from the Atherosclerosis Risk in Communities (ARIC) study have shown that increased levels of t-PA and PAI-1 are associated with subclinical carotid atherosclerosis, measured as increased intima-media thickness [44].

Therefore, different proteins involved in haemostasis and synthesized by endothelial cells have been proposed as markers of endothelial dysfunction. The main problem is the relatively poor specificity of these markers, which may hamper their use in detection or monitoring of endothelial dysfunction.

\section{Adhesion Molecules}

Binding of circulating leukocytes to the vascular endothelium and further leukocyte migration into the subendothelial space are major processes in the development of atherosclerosis. These events are mediated through diverse cellular adhesion molecules that are expressed on the 
surface of vascular endothelial cells, namely vascular cell adhesion molecule-1 (VCAM-1), intercellular adhesion molecule-1 (ICAM-1), E-selectin and P-selectin. Circulating soluble forms of these adhesion molecules have been detected in plasma [45].

Several studies have suggested that circulating adhesion molecules may serve as markers of endothelial damage and/or atherosclerosis. In the ARIC study it was shown that E-selectin and ICAM-1 are associated with the carotid artery intima-media thickness and are independent predictors of incident coronary artery disease [46]. This study also showed higher levels of ICAM-1 in smokers. Squadrito and coworkers observed higher levels of circulating ICAM-1 and E-selectin in patients with acute myocardial infarction. Increased levels of plasma P-selectin have been observed in unstable angina, myocardial infarction and hypercholesterolemia [48]. Furthermore, ICAM-1 levels may predict cardiovascular event rates in apparently healthy men [49].

Of the risk factors, serum lipoproteins seem to be especially related to the levels of circulating adhesion molecules. In the study by Hackman and co-workers, dyslipidaemia was associated with elevated levels of ICAM1, VCAM-1, and E-selectin [50]. Successful therapy with lipid-lowering statins significantly reduced the levels of circulating E-selectin [51, 52]. Therefore, these studies suggested that cell adhesion molecules may serve as potential markers of the efficacy of lipid-lowering therapy.

Endothelial function may be tested noninvasively in the peripheral conduit arteries using high-resolution external vascular ultrasonography. In this non-invasive method, arterial diameter is measured in response to an increase in shear stress, which causes endothelium-dependent dilatation. The brachial arterial dilation response to increased blood flow during reactive hyperaemia has been shown to be mainly caused by endothelial release of NO, and to correlate significantly with coronary endothelial function [53].

Endothelial dysfunction has been demonstrated in subjects with different risk factors of atherosclerosis, such as hypercholesterolemia, diabetes, hypertension, smoking and in patients with atherosclerotic disease (coronary, peripheral arterial). There also exists close relationship between the intensity of an individual risk factor or the number of presented risk factors, and endothelial function.

A large body of evidence indicates that endothelial dysfunction is a characteristic of patients with essential hypertension [54-56]. Endothelial dysfunction associated with essential hypertension is characterized by impaired NO availability, determined by oxidative stress production, which causes NO breakdown [57]. In patients with essential hypertension endothelium-dependent vasodilation is impaired to acetylcholine and bradykinin application. These findings indicate that the endothelial dysfunction in this condition is not related to a specific defect of a single intracellular signal-transduction pathway and suggests a more generalized abnormality of endothelial vasodilator function. These results are in contrast with those is hypercholesterolemic patients in whom response only to acetylcholine is impaired [58].

It is presently unclear whether endothelial dysfunction is a primary abnormality or a consequence of the elevated blood pressure values [59]. Data in animal models of genetic hypertension are in favour of direct damage of the endothelial cells secondary to a prolonged increase of the haemodynamic load and shear stress. The endothelial dysfunction observed in hypertension appears to be a consequence of high blood pressure since a variety of antihypertensive treatment normalizes this responses. However, in normotensive offspring's of hypertensive parents, an early alteration of endothelium-dependent vasodilation in the forearm was observed [60]. We also found impaired endothelial function in offspring's of parents with essential hypertension although their blood pressure was in normal range [61]. These data suggest a possibly relevant role of genetic mechanisms in the genesis of endothelial dysfunction in hypertension.

Evidence that endothelial dysfunction occurs in diabetic patients is found an elevated plasma concentration of the von-Willebrand factor, impaired prostacyclin and plasminogen activator release, and decreased lipoprotein lipase activity. It has also been reported that in patients with insulin-dependent and insulin-independent diabetes mellitus some aspects of endothelial dysfunction related to vasodilator response are present $[62,63]$. It was shown that flow mediated endothelial vasodilation capability of peripheral arteries is closely related to microalbuminuria $[64$, 65]. It is possible that endothelial dysfunction contributes to the overt vascular complication in diabetes. A considerable body of evidence indicates that endothelial dysfunction is closely associated with a development of diabetic retinopathy, nephropathy and is involved in pathogenesis of accelerated forms of atherosclerosis in diabetic patients [66]. In some patients it is preserved regardless of disease duration, provided urinary albumin excretion is normal. There is a substantial evidence that endothelium-dependent vasodilation is normal in both conduit arteries and resistance vessels of diabetic animals. In patients with insulindependent diabetes mellitus the abnormal endothelium dependent vasodilation has been shown to be caused by decreased release or activity of NO.

\section{Involvement of Endothelial Dysfunction in Atherogenesis}

Endothelial dysfunction is the first step of atherosclerosis and precedes macroscopic morphologic alteration. Endothelial dysfunction has been demonstrated in subjects with different risk factors of atherosclerosis, such as hypercholesterolemia [67, 68], diabetes [69, 70], hypertension [71, 72], smoking [73] and in patients with atherosclerotic disease (coronary, peripheral arterial) [74-77]. We demonstrated that endothelial dysfunction progresses with the duration of hypertension, diabetes or smoking and that a close relationship exists between the intensity of an individual risk factor or the number of risk factors present and endothelial function $[61,70]$. Furthermore, treatment of risk factors results in improvement of endothelial dysfunction. It has been shown that treatment of hypercholesterolemia with different statins improves endothelial function [78]. Regression of endothelial dysfunction was observed during treatment of arterial hypertension with various drugs and during physical training of patients with cardiac insufficiency and metabolic syndrome [79]. We also observed improvement of endothelial dysfunction during growth hormone replacement in growth hormone-deficient patients 
[80]. Therefore, a dose-response relation exists between risk factors of atherosclerosis and endothelial dysfunction.

\section{Endothelial dysfunction And Cardiovascular Risk}

Impairment of the endothelium-dependent dilation has been found to predict long-term coronary events in patients with or without obstructive CAD $[81,82]$. The treatment of risk factors and vascular conditions associated with endothelial dysfunction has been shown to improve longterm prognosis. This is probably due to "stabilization" of non-obstructive atherosclerotic plaques and improvement to endothelium vasomotor response [83]. These observations support the concept of "reversibility" of the atherosclerotic functional changes by specific interventions targeting endothelial dysfunction. Thus, the key points for prevention of $\mathrm{CV}$ events could be early identification of endothelial dysfunction using non-invasive tests in subjects at risk and the treatment of risk factors.

\section{INTIMA-MEDIA THICKNESS}

In atherogenesis arterial wall morphological changes occur during a presumably long subclinical lag phase characterized by gradual thickening of intima. The measurement of intima-media thickness of the large superficial arteries, especially the carotid, using high resolution B-mode ultrasonography has emerged as one of the methods of choice for determining the anatomical extent of arterial wall deterioration and for assessing cardiovascular risk [84]. Population studies have shown a strong correlation between carotid IMT and several cardiovascular risk factors and it has also been found IMT to be associated with the extent of atherosclerosis and end-organ damage of high risk patients [85-87].

Both the carotid and femoral IMTs increased significantly with age, and the IMT was shown to be greater in men than in women. The carotid IMT was estimated to increase by $0.04 \mathrm{~mm}$ for every 10 years [85]. These findings were also supported by the EAS (The Edinburgh Artery Study), which showed a continuous increase in carotid IMT with age [88].

Carotid and femoral IMT is also influenced by cholesterol level. IMT is most notably increased in patients with familial hypercholesterolemia and its increase was already found to be significant in young patients with familial hypercholesterolemia $[89,90]$. Of all traditional risk factors, hypertension appears to have the greatest effect on IMT.

Further, carotid IMT is affected by lifestyle. In the Monitored Atherosclerosis Regression Study (MARS) [91] cholesterol intake, body mass index and smoking were significantly related to the annual progression of carotid IMT. Cigarette smoking has also been shown to increase the IMT in both the carotid and femoral arteries [73]. New risk factors have also been tested with regard to their relationship to carotid IMT. Some of these studies have demonstrated a consistent association of these risk factors with increased IMT, such as lipoprotein-a [92].

\section{Carotid IMT, Cardiovascular Alterations and Clinical Events}

Carotid IMT is a well-established measure for assessing the degree of atherosclerosis [93] which has also been found to be associated with cardiovascular alterations or organ damage [94, 95]. It was shown that increased IMT is related to brain white matter lesions, angiographically assessed coronary artery disease and the extent of coronary artery calcification [96]. In the Insulin Resistance Atherosclerosis Study (IRAS) an interrelationship was found between carotid IMT and microalbuminuria in non-diabetic and non-insulin dependent diabetes mellitus subjects [97]. Different studies demonstrated that increased carotid IMT is associated with the prevalence of peripheral arterial occlusive disease and with the prevalence of abdominal aortic aneurysm.

All these studies are concordant in demonstrating that increased IMT is a powerful predictor of coronary and cerebrovascular events, whatever the method and the site of measurement, including the common carotid far wall IMT.

\section{Usefulness of IMT Measurement}

Increased carotid IMT is a mirror of the atherosclerotic burden and a predictor of subsequent events. Because of its quantitative value, carotid IMT measurement is more and more frequently used in clinical trials to test the effects of different preventive measures, including drugs. More recently, there has been interest in the clinical use of this technique for detecting preclinical (asymptomatic) atherosclerosis and for identifying subjects at high risk. Measurement of carotid IMT could influence a clinician to intervene with medication and to use more aggressive treatment of risk factors in primary prevention, and in patients with atherosclerotic disease in whom there is evidence of progression and extension of atherosclerotic disease. IMT measurement obtained by ultrasonography correlates with pathology measurements and the reproducibility of this technique is good $[98,99]$. For more extensive use of this method in clinical practice, a consensus concerning the standardization of methods of measurement and precise definition of the threshold between normal and pathological IMT values is urgently needed.

\section{CONCLUSIONS}

The development of atherosclerosis is related to risk factors. However, atherosclerosis frequently appears also in subjects without classical risk factors of atherosclerosis (in $30-40 \%$ ). Therefore, in recent years research on atherosclerosis has been mainly focused toward identifying a new non-classical risk factors and markers of early preclinical atherosclerosis. The identification of the disease in its early - asymptomatic phase - is very important from prognostic and preventive aspects. Detection of early preclinical - atherosclerosis lesions is of utmost importance for identification of individuals in whom atherosclerotic process is already present, and represents high risk for cardiovascular events. 
Most frequently investigated indicators of atherosclerosis are: circulating (blood) markers, functional deterioration and morphologic alteration of arterial wall. Morphologic changes of arterial wall were shown to be the most valuable indicators of prognosis and predictor of cardiovascular events. Measurement of intima media thickness is simple and most reproducible technique that enables quantifying of harmful effects of risk factors on arterial wall, and positive effects of their management. It also represents the most standardized tool for detection of the earliest morphologic deterioration of arterial wall in atherosclerosis.

Endothelial dysfunction is the earliest in-vivo measurable functional alteration of arterial wall in atherogenesis and most probably represents common mechanism of the damage of the vessel wall caused by different risk factors. However, its measurement is time-consuming and its reproducibility needs to be improved. Larger studies are needed to demonstrate clinical utility of these techniques.

Circulating markers of atherosclerosis are related to increased vascular risk. For clinical purposes, some inflammatory biomarkers, particularly CRP is most promising. In last decades, research has been mainly focused toward identifying circulating markers of plaques stability and instable coronary patients.

Determination of markers of preclinical atherosclerosis could be useful in detection of individuals at highest risk who need more aggressive treatment of risk factors of atherosclerosis.

\section{REFERENCES}

[1] Ross R. The pathogenesis of atherosclerosis: a perspective for the 1990s. Nature 1993; 362: 801-9.

[2] Wattanakit K, Folsom AR, Chambless LE, Nieto FJ. Risk factors for cardiovascular event recurrence in the Atherosclerosis Risk in Communities (ARIC) study. Am Heart J 2005; 149: 606-12.

[3] Konstantino Y, Wolk R, Terra SG, Nguyen TT, Fryburg DA. Nontraditional biomarkers of atherosclerosis in stable and unstable coronary artery disease, do they differ? Acute Card Care 2007; 9: 197-206.

[4] Libby P, Ridker PM, Maseri A. Inflammation and atherosclerosis. Circulation 2002; 105: 1135-43.

[5] Ridker PM, Hennekens CH, Buring JE, Rifai N. C-reactive protein and other markers of inflammation in the prediction of cardiovascular disease in women. N Engl J Med 2000; 342: 836-43.

[6] Ridker PM. High-sensitivity C-reactive protein: potential adjunct for global risk assessment in the primary prevention of cardiovascular disease. Circulation 2001; 103: 1813-8.

[7] Fukumoto Y, Libby P, Rabkin E, et al. Statins alter smooth muscle cell accumulation and collagen content in established atheroma of watanabe heritable hyperlipidemic rabbits. Circulation 2001; 103: 993-9.

[8] Ridker PM, Rifai N, Clearfield M, et al. Measurement of Creactive protein for the targeting of statin therapy in the primary prevention of acute coronary events. N Engl J Med 2001; 344: 1959-65.

[9] Ridker PM, Danielson E, Fonseca FA, et al. Reduction in Creactive protein and LDL cholesterol and cardiovascular event rates after initiation of rosuvastatin: a prospective study of the JUPITER trial. Lancet 2009; 373: 1175-82.

[10] Ridker PM, Buring JE, Rifai N, Cook NR. Development and validation of improved algorithms for the assessment of global cardiovascular risk in women: the Reynolds Risk Score JAMA 2007; 297: 611-9.

[11] Tracy RP, Arnold AM, Ettinger W, et al. The relationship of fibrinogen and factors VII and VIII to incident cardiovascular disease and death in the elderly: results from the cardiovascular health study. Arterioscler Thromb Vasc Biol 1999; 19: 1776-83.
[12] Von der ThĂLsen JH, Kuiper J, Van Berkel TJC, Biessen EAL. Interleukins in Atherosclerosis: Molecular Pathways and Therapeutic Potential. Pharmacological Reviews 2003; 55: 133-66.

[13] Huber SA, Sakkinen P, David C, Newell MK, Tracy RP. T helpercell phenotype regulates atherosclerosis in mice under conditions of mild hypercholesterolemia. Circulation 2001; 103: 2610-6.

[14] Erzen B, Sabovic M, Sebestjen M, Keber I, Poredos P. Interleukin6 correlates with endothelial dysfunction in young post-myocardial infarction patients. Cardiology 2007; 107: 111-6.

[15] Loria V, Dato I, Graziani F, Biasucci LM. Myeloperoxidase: a new biomarker of inflammation in ischemic heart disease and acute coronary syndromes. Mediators Inflamm 2008; 2008: 135625.

[16] Baldus S, Heeschen C, Meinertz T, et al. Myeloperoxidase serum levels predict risk in patients with acute coronary syndromes. Circulation 2003; 108: 1440-5.

[17] Brennan ML, Penn MS, Van Lente F, et al. Prognostic value of myeloperoxidase in patients with chest pain. N Engl J Med 2003; 349: 1595-604.

[18] Blankenberg S, Rupprecht HJ, Poirier O, et al. Plasma concentrations and genetic variation of matrix metalloproteinase 9 and prognosis of patients with cardiovascular disease. Circulation 2003; 107: 1579-85.

[19] Squire IB, Evans J, Ng LL, Loftus IM, Thompson MM. Plasma MMP-9 and MMP-2 following acute myocardial infarction in man: correlation with echocardiographic and neurohumoral parameters of left ventricular dysfunction. J Card Fail 2004; 10: 328-33.

[20] Szmitko PE, Wang CH, Weisel RD, et al. New markers of inflammation and endothelial cell activation: Part I. Circulation 2003; 108: 1917-23.

[21] Antoniades C, Bakogiannis C, Tousoulis D, Antonopoulos AS, Stefanadis C. The CD40/CD40 Ligand System: Linking Inflammation With Atherothrombosis. J Am Coll Cardiol 2009; 54: 669-77.

[22] Varo N, de Lemos JA, Libby P, et al. Soluble CD40L: risk prediction after acute coronary syndromes. Circulation 2003; 108 : 1049-52.

[23] Apple FS, Wu AHB, Mair J, et al. Future Biomarkers for Detection of Ischemia and Risk Stratification in Acute Coronary Syndrome. Clin Chem 2005; 51: 810-24.

[24] Antoniades C, Bakogiannis C, Tousoulis D, Antonopoulos AS, Stefanadis C. The CD40/CD40 ligand system: linking inflammation with atherothrombosis. J Am Coll Cardiol 2009; 54: 669-77.

[25] Martinez A, Januzzi JL. Novel markers for the evaluation of patients with suspected ischemic heart disease. Point Care 2006; 5: 28-37.

[26] Oliver MF, Opie LH. Effects of glucose and fatty acids on myocardial ischaemia and arrhythmias. Lancet 1994; 343: 155-8.

[27] Tjwa M, Luttun A, Autiero M, Carmeliet P. VEGF and PlGF: two pleiotropic growth factors with distinct roles in development and homeostasis. Cell Tissue Res 2003; 314: 5-14.

[28] Hattori K, Heissig B, Wu Y, et al. Placental growth factor reconstitutes hematopoiesis by recruiting VEGFR1(+) stem cells from bone-marrow microenvironment. Nat Med 2002; 8: 841-9.

[29] Heeschen C, Dimmeler S, Fichtlscherer S, et al. Prognostic value of placental growth factor in patients with acute chest pain. JAMA 2004; 291: 435-41.

[30] Drexler H. Factors involved in the maintenance of endothelial function. Am J Cardiol 1998; 82: 3S-4S.

[31] Dimmeler S, Fleming I, Fisslthaler B, et al. Activation of nitric oxide synthase in endothelial cells by Akt-dependent phosphorylation. Nature 1999; 399: 601-5.

[32] Garcin ED, Arvai AS, Rosenfeld RJ, et al. Anchored plasticity opens doors for selective inhibitor design in nitric oxide synthase. Nat Chem Biol 2008; 4: 700-7.

[33] Lowenstein CJ, Michel T. What's in a name? eNOS and anaphylactic shock. J Clin Invest 2006; 116: 2075-8.

[34] Michel T, Feron O. Nitric oxide synthases: which, where, how, and why? J Clin Invest 1997; 100: 2146-52.

[35] Anggrahini DW, Emoto N, Nakayama K, et al. Vascular endothelial cell-derived endothelin-1 mediates vascular inflammation and neointima formation following blood flow cessation. Cardiovasc Res 2009; 82: $143-51$.

[36] Dimitrijevic I, Ekelund U, Edvinsson ML, Edvinsson L. Increased expression of endothelin ET(B) and angiotensin AT(1) receptors in 
peripheral resistance arteries of patients with suspected acute coronary syndrome. Heart Vessels 2009; 24: 393-8.

[37] Lerman A, Edwards BS, Hallett JW, et al. Circulating and tissue endothelin immunoreactivity in advanced atherosclerosis. $\mathrm{N}$ Engl $\mathrm{J}$ Med 1991; 325: 997-1001.

[38] Blann AD, Davis A, Miller JP, McCollum CN. Von Willebrand factor and soluble E-selectin in hyperlipidaemia: relationship to lipids and vascular disease. Am J Hematol 1997; 55: 15-23.

[39] Spiel AO, Gilbert JC, Jilma B. Von willebrand factor in cardiovascular disease: focus on acute coronary syndromes. Circulation 2008; 117: 1449-59.

[40] Miura M, Kaikita K, Matsukawa M, et al. Prognostic value of plasma von Willebrand factor-cleaving protease (ADAMTS13) antigen levels in patients with coronary artery disease. Thromb Haemost 103: 623-9.

[41] Paulinska P, Spiel A, Jilma B. Role of von Willebrand factor in vascular disease. Hamostaseologie 2009; 29: 32-8.

[42] Cortellaro M, Boschetti C, Cofrancesco E, et al. The PLAT Study: hemostatic function in relation to atherothrombotic ischemic events in vascular disease patients. Principal results. PLAT Study Group. Progetto Lombardo Atero-Trombosi (PLAT) Study Group. Arterioscler Thromb 1992; 12: 1063-70.

[43] Geppert A, Graf S, Beckmann R, et al. Concentration of endogenous tPA antigen in coronary artery disease: relation to thrombotic events, aspirin treatment, hyperlipidemia, and multivessel disease. Arterioscler Thromb Vasc Biol 1998; 18: 1634-42.

[44] Salomaa V, Stinson V, Kark JD, et al. Association of fibrinolytic parameters with early atherosclerosis. The ARIC Study. Atherosclerosis Risk in Communities Study. Circulation 1995; 91 : 284-90.

[45] Pigott R, Dillon LP, Hemingway IH, Gearing AJ. Soluble forms of E-selectin, ICAM-1 and VCAM-1 are present in the supernatants of cytokine activated cultured endothelial cells. Biochem Biophys Res Commun 1992; 187: 584-9.

[46] Hwang S-J, Ballantyne CM, Sharrett AR, et al. Circulating Adhesion Molecules VCAM-1, ICAM-1, and E-selectin in Carotid Atherosclerosis and Incident Coronary Heart Disease Cases : The Atherosclerosis Risk In Communities (ARIC) Study. Circulation 1997; 96: 4219-25.

[47] Altavilla D, Squadrito F, Ammendolia L, et al. Monocytes and lymphocytes as active participants in the pathogenesis of experimental shock. Inflamm Res 1996; 45: 398-404.

[48] Xu DY, Zhao SP, Peng WP. Elevated plasma levels of soluble Pselectin in patients with acute myocardial infarction and unstable angina. An inverse link to lipoprotein(a). Int J Cardiol 1998; 64: 253-8.

[49] Ridker PM, Hennekens CH, Roitman-Johnson B, Stampfer MJ, Allen J. Plasma concentration of soluble intercellular adhesion molecule 1 and risks of future myocardial infarction in apparently healthy men. Lancet 1998; 351: 88-92.

[50] Hackman A, Abe Y, Insull W, Jr., et al. Levels of soluble cell adhesion molecules in patients with dyslipidemia. Circulation 1996; 93: 1334-8

[51] Porta B, Baldassarre D, Camera M, et al. E-selectin and TFPI are associated with carotid intima-media thickness in stable IHD patients: The baseline findings of the MIAMI study. Nutrition, metabolism, and cardiovascular diseases: Nutr Metab Cardiovasc Dis 2008; 18: 320-28.

[52] Peter M, Guy JF, Heidi D, et al. Reduction of soluble P-selectin by statins is inversely correlated with the progression of coronary artery disease. Int J Cardiol 2006; 106: 183-90.

[53] Raitakari OT, Celermajer DS. Testing for endothelial dysfunction. Ann Med 2000; 32: 293-304.

[54] Panza JA, Quyyumi AA, Brush JE, Jr., Epstein SE. Abnormal endothelium-dependent vascular relaxation in patients with essential hypertension. N Engl J Med 1990; 323: 22-7.

[55] Ouvina SM, La Greca RD, Zanaro NL, Palmer L, Sassetti B. Endothelial dysfunction, nitric oxide and platelet activation in hypertensive and diabetic type II patients. Thromb Res 2001; 102: 107-14.

[56] Mombouli JV, Vanhoutte PM. Endothelial dysfunction: from physiology to therapy. J Mol Cell Cardiol 1999; 31: 61-74.

[57] Taddei S, Virdis A, Ghiadoni L, Magagna A, Salvetti A. Vitamin C improves endothelium-dependent vasodilation by restoring nitric oxide activity in essential hypertension. Circulation 1998; 97: 2222-9.
[58] Taddei S, Salvetti A. Endothelial dysfunction in essential hypertension: clinical implications. J Hypertens 2002; 20: 1671-74.

[59] Rizzoni D. Endothelial dysfunction in hypertension: fact or fantasy? J Hypertens 2002; 20: 1479-81.

[60] Taddei S, Virdis A, Mattei P, et al. Defective L-arginine-nitric oxide pathway in offspring of essential hypertensive patients. Circulation 1996; 94: 1298-303.

[61] Zizek B, Poredos P, Videcnik V. Endothelial dysfunction in hypertensive patients and in normotensive offspring of subjects with essential hypertension. Heart 2001; 85: 215-7.

[62] Clarkson P, Celermajer DS, Donald AE, et al. Impaired vascular reactivity in insulin-dependent diabetes mellitus is related to disease duration and low density lipoprotein cholesterol levels. J Am Coll Cardiol 1996; 28: 573-9.

[63] Schiekofer S, Balletshofer B, Andrassy M, Bierhaus A, Nawroth PP. Endothelial dysfunction in diabetes mellitus. Semin Thromb Hemost 2000; 26: 503-11.

[64] Yudkin JS, Forrest RD, Jackson CA. Microalbuminuria as predictor of vascular disease in non-diabetic subjects. Islington Diabetes Survey. Lancet 1988; 2: 530-3.

[65] Poredos P, Kek A. Relation of blunted dilation of the brachial artery in insulin-dependent diabetes mellitus to microalbuminuria. Am J Cardiol 2000; 86: 364-67.

[66] Lim SC, Caballero AE, Smakowski P, et al. Soluble intercellular adhesion molecule, vascular cell adhesion molecule, and impaired microvascular reactivity are early markers of vasculopathy in type 2 diabetic individuals without microalbuminuria. Diabetes Care 1999; 22: 1865-70

[67] Pirro M, Bagaglia F, Paoletti L, Razzi R, Mannarino MR. Hypercholesterolemia-associated endothelial progenitor cell dysfunction. Ther Adv Cardiovasc Dis 2008; 2: 329-39.

[68] Landmesser U, Hornig B, Drexler H. Endothelial dysfunction in hypercholesterolemia: mechanisms, pathophysiological importance, and therapeutic interventions. Semin Thromb Hemost 2000; 26: 529-37.

[69] Olsen MH, Andersen UB, Wachtell K, Ibsen H, Dige-Petersen H. A possible link between endothelial dysfunction and insulin resistance in hypertension. A LIFE substudy. Losartan Intervention For Endpoint-Reduction in Hypertension. Blood Press 2000; 9: 132-9.

[70] Poredos P, Kek A. Relation of blunted dilation of the brachial artery in insulin-dependent diabetes mellitus to microalbuminuria. Am J Cardiol 2000; 86: 364-7.

[71] Schulz E, Jansen T, Wenzel P, Daiber A, Munzel T. Nitric oxide, tetrahydrobiopterin, oxidative stress, and endothelial dysfunction in hypertension. Antioxid Redox Signal 2008; 10: 1115-26.

[72] Taddei S, Virdis A, Ghiadoni L, Salvetti G, Salvetti A. Endothelial dysfunction in hypertension. J Nephrol 2000; 13: 205-10.

[73] Poredos P, Orehek M, Tratnik E. Smoking is associated with doserelated increase of intima-media thickness and endothelial dysfunction. Angiology 1999; 50: 201-8.

[74] Vanhoutte PM. Endothelial dysfunction: the first step toward coronary arteriosclerosis. Circ J. 2009; 73: 595-601.

[75] Schachinger V, Zeiher AM. Atherosclerosis-associated endothelial dysfunction. Z Kardiol 2000; 89(Suppl 9): IX/70-4.

[76] Shimokawa H. Primary endothelial dysfunction: atherosclerosis. J Mol Cell Cardiol 1999; 31: 23-37.

[77] Pitkanen OP, Raitakari OT, Niinikoski H, et al. Coronary flow reserve is impaired in young men with familial hypercholesterolemia. J Am Coll Cardiol 1996; 28: 1705-11.

[78] Vogel RA. Cholesterol lowering and endothelial function. Am J Med 1999; 107: 479-87.

[79] Poredos P, Kek A, Verhovec R. Morphological and functional changes of the arterial wall in subjects at risk of atherosclerosis and in patients with peripheral arterial occlusive disease. Vasa 1997; 26: 271-6.

[80] Pfeifer M, Verhovec R, Zizek B, et al. Growth hormone (GH) treatment reverses early atherosclerotic changes in $\mathrm{GH}$-deficient adults. J Clin Endocrinol Metab 1999; 84: 453-7.

[81] Halcox JP, Schenke WH, Zalos G, et al. Prognostic value of coronary vascular endothelial dysfunction. Circulation 2002; 106 : 653-8.

[82] Neunteufl T, Heher S, Katzenschlager R, et al. Late prognostic value of flow-mediated dilation in the brachial artery of patients with chest pain. Am J Cardiol 2000; 86: 207-10. 
[83] Bonetti PO, Lerman LO, Lerman A. Endothelial dysfunction: a marker of atherosclerotic risk. Arterioscler Thromb Vasc Biol 2003; 23: 168-75.

[84] Kanters SD, Algra A, van Leeuwen MS, Banga JD. Reproducibility of in vivo carotid intima-media thickness measurements: a review. Stroke 1997; 28: 665-71.

[85] Ando F, Takekuma K, Niino N, Shimokata H. Ultrasonic evaluation of common carotid intima-media thickness (IMT)-influence of local plaque on the relationship between IMT and age. J Epidemiol 2000; 10: S10-7.

[86] Salonen R, Tervahauta M, Salonen JT, et al. Ultrasonographic manifestations of common carotid atherosclerosis in elderly eastern Finnish men. Prevalence and associations with cardiovascular diseases and risk factors. Arterioscler Thromb 1994; 14: 1631-40.

[87] Mancini GB. Carotid intima-media thickness as a measure of vascular target organ damage. Curr Hypertens Rep 2000; 2: 71-7.

[88] Mowbray PI, Lee AJ, Fowkes GR, Allan PL. Cardiovascular risk factors for early carotid atherosclerosis in the general population: the Edinburgh Artery Study. J Cardiovasc Risk 1997; 4: 357-62.

[89] Gariepy J, Simon A, Massonneau M, Linhart A, Levenson J. Wall thickening of carotid and femoral arteries in male subjects with isolated hypercholesterolemia. PCVMETRA Group. Prevention Cardio-Vasculaire en Medecine du Travail. Atherosclerosis 1995; 113: 141-51.

[90] Lavrencic A, Kosmina B, Keber I, Videcnik V, Keber D. Carotid intima-media thickness in young patients with familial hypercholesterolaemia. Heart 1996; 76: 321-5.

[91] Hodis HN. Reversibility of atherosclerosis-evolving perspectives from two arterial imaging clinical trials: the cholesterol lowering atherosclerosis regression study and the monitored atherosclerosis regression study. J Cardiovasc Pharmacol 1995; 25 Suppl 4: S2531 .

[92] Schreiner PJ, Heiss G, Tyroler HA, et al. Race and gender differences in the association of $\mathrm{Lp}(\mathrm{a})$ with carotid artery wall thickness. The Atherosclerosis Risk in Communities (ARIC) Study. Arterioscler Thromb Vasc Biol 1996; 16: 471-8.

[93] Salonen JT, Salonen R. Ultrasound B-mode imaging in observational studies of atherosclerotic progression. Circulation 1993; 87: II56-65.

[94] Manios E, Tsivgoulis G, Koroboki E, et al. Impact of prehypertension on common carotid artery intima-media thickness and left ventricular mass. Stroke 2009; 40: 1515-8.

[95] Davis PH, Dawson JD, Mahoney LT, Lauer RM. Increased carotid intimal-medial thickness and coronary calcification are related in young and middle-aged adults. The Muscatine study. Circulation 1999; 100: 838-42.

[96] Bots ML, van Swieten JC, Breteler MM, et al. Cerebral white matter lesions and atherosclerosis in the Rotterdam Study. Lancet 1993; 341: 1232-7.

[97] Mykkanen L, Zaccaro DJ, O'Leary DH, et al. Microalbuminuria and carotid artery intima-media thickness in nondiabetic and NIDDM subjects. The Insulin Resistance Atherosclerosis Study (IRAS). Stroke 1997; 28: 1710-6.

[98] Executive Summary of The Third Report of The National Cholesterol Education Program (NCEP) Expert Panel on Detection, Evaluation, And Treatment of High Blood Cholesterol In Adults (Adult Treatment Panel III). JAMA 2001; 285: 2486-97.

[99] Loizou CP, Pattichis CS, Nicolaides AN, Pantziaris M. Manual and automated media and intima thickness measurements of the common carotid artery. IEEE Trans Ultrason Ferroelectr Freq Control 2009; 56: 983-94.

(C) Pavel Poredos; Licensee Bentham Open

This is an open access article licensed under the terms of the Creative Commons Attribution Non-Commercial License (http://creativecommons.org/licenses/by$\mathrm{nc} / 3.0 /$ ) which permits unrestricted, non-commercial use, distribution and reproduction in any medium, provided the work is properly cited. 